
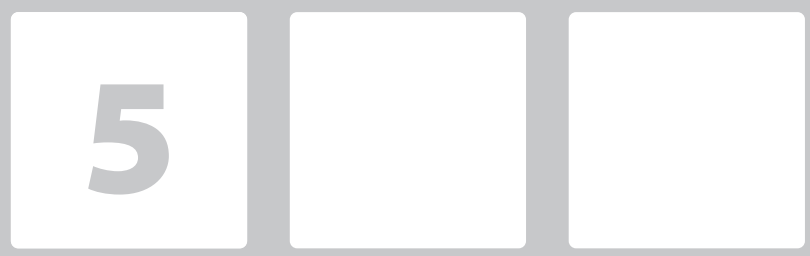

\title{
SATISFAÇÃO E MOTIVAÇÃO NO TRABALHO: INSIGHTS SOBRE PERCEPÇÃO DA JUSTIÇA DISTRIBUTIVA E REMUNERAÇÃO ESTRATÉGICA POR INDIVÍDUOS DA GERAÇÃO Z
}

\section{Satisfaction and Motivation at Work: Insights About the Perception of Distributive Justice and Strategic Remuneration by Individuals of Generation Z}

\section{Silvia Spagnol Simi dos Santos}

Universidade do Oeste de Santa Catarina - UNOESC email: silvia.simi@unoesc.edu.br

\section{Ana Maria Olivo}

Universidade do Oeste de Santa Catarina - UNOESC

email:anamaria.olivo@hotmail.com

\section{David Rodrigo Petry}

Universidade do Oeste de Santa Catarina - UNOESC email:david.petry@unoesc.edu.br

\section{leda Margarete Oro}

Universidade do Oeste de Santa Catarina - UNOESC

email:ieda.oro@unoesc.edu.br

\section{RESUMO}

A justiça organizacional é assunto contemporâneo que passou a ser observado pelas organizações em função da Geração Z. Este estudo objetiva verificar a relação existente entre justiça distributiva, remuneração estratégica e os fatores de satisfação e motivação de indivíduos da Geração Z no mercado de trabalho. Como metodologia optou-se pela abordagem quantitativa por intermédio de survey online enviado para 13.000 indivíduos pertencentes a essa geração, resultando em uma amostra de 1.112 pessoas. A análise dos resultados foi realizada com utilização do software SPSS ${ }^{\circledR}$ para análise fatorial e $\mathrm{AMOS}^{\circledR}$ para a modelagem de equações estruturais. A partir das hipóteses testadas foi possível verificar que a percepção de Justiça Distributiva e da Remuneração Estratégica influenciam na Motivação e na Satisfação de indivíduos da Geração Z no mercado de trabalho. Os achados revelam que não é o tipo de remuneração que é o mais importante, mas sim a percepção de ganhos.

Palavras-Chaves: Remuneração Estratégica, Justiça distributiva, Motivação, Satisfação, Geração Z.

\section{ABSTRACT}

Organizational justice is a contemporary issue that has come to be seen by Generation Z functions. This study aims to verify an existing relationship between distributed justice, strategic statistics, and the factors of satisfaction and motivation of people generated by $Z$ in the job market. As the methodology opted for the quantitative approach through an online survey sent to 13,000 individuals belonging to this generation, resulting in a sample of 1,112 people. An analysis of the results was performed using the SPSS ${ }^{\circledR}$ software for factor analysis and AMOS ${ }^{\circledR}$ for modeling selected equations. From the tested hypotheses, it was possible to verify the perception of Distribution Justice and Strategic Remuneration that influences Motivation and Satisfaction of Generation Z Indications in the job market. The findings reveal that it is not the type of remuneration that is most important, but the perception of earnings.

Keywords: Strategic Remuneration, Distributive Justice, Motivation, Satisfaction, Generation Z. 


\section{INTRODUÇÃO}

A justiça organizacional é tema imperativo em organizações que buscam pela satisfação e retenção de seus talentos. A remuneração estratégica é uma das formas de estimular a busca pela excelência e superação dos colaboradores como recompensa pelo seu desempenho. Entretanto, o alcance dos objetivos e metas não pode perder o foco com insatisfação ou rotatividade de seus recursos humanos. Nesse contexto, a justiça organizacional é hoje uma das questões mais importantes e valiosas da gestão de recursos humanos (Ahmad e Abdolkhalegh, 2015).

Ao encontro desta necessidade organizacional, estudos como o de Palozolli (2000), Assmar, Ferreira e Souto (2005), Sampaio (2009), Camino e Roazzi (2009), Tadeucci (2009), Gheno e Berlitz (2011) e Ahmad e Abdolkhalegh (2015) buscaram explicar como a percepção da justiça distributiva reflete na motivação dos membros da organização, ao sentirem se esta distribui seus recursos (salários, premiações, punições, etc.) de maneira justa e igualitária.

No entanto, a partir dos anos 2000, a exigência do mercado de trabalho por resultados mais rápidos demonstrou a necessidade de compreender a inserção de jovens com novo perfil de trabalho, conhecida como indivíduos da Geração Z. Esta geração, composta por indivíduos nascidos a partir de 1989, até então pouco valorizada pelas organizações (CERETTA; FROEMMING, 2011), emerge, a partir de 2012, como alvo estratégico e necessário no cotidiano das organizações, tendo em vista que compõem a maior massa de mão de obra disponível no mercado. São chamados ainda de geração digital, on-line, conectada, silenciosa, pontocom, entre outras (VEIGA NETO; SOUZA; ALMEIDA; CASTRO; BRAGA JUNIOR, 2015).

A Geração Z consolidou-se pelas características intrínsecas e comportamentais como desejo de liberdade, inovação e colaboração (TAPSCOTT, 2010), peculiarmente familiarizadas com a tecnologia da informação (JACQUES et al., 2015), além da rapidez com que conseguem as informações e a fácil irritabilidade quando estas não lhes chegam rapidamente (VEIGA NETO et al., 2015). Todos estes aspectos, distintos de gerações anteriores (como por exemplo veteranos, Baby Boomers, Geração X e Geração Y), estão exigindo das organizações significativas mudanças na maneira como ocorrem as relações funcionais.

Surge neste contexto, uma característica diferenciada de retorno aos colaboradores que apresentam diferenciação e desempenho produtivo. Entre os fatores motivacionais de desempenho está a remuneração. Aliar a remuneração justa com a filosofia da organização é um paradigma (TRINDADE; CUNHA; NUNES; SANTOS, 2011). Este formato diferenciado e particular de cada organização, pode ser teoricamente conceituado como Remuneração Estratégica, o qual decorre de fatores de sucesso das estratégias de recursos humanos para agregar valor aqueles que contribuem e geram resultados positivos à organização (ZAMBRANO; MERINO; CASTELLANOS, 2012) .

Diante disso, a questão central desta investigação é: para os indivíduos da Geração Z, a justiça distributiva e a remuneração estratégica propiciam motivação e satisfação no trabalho? Objetiva-se, portanto, verificar a relação entre a justiça distributiva, a remuneração estratégica, os fatores motivacionais e de satisfação no mercado de trabalho de indivíduos da Geração Z. Para tanto, utilizou-se procedimentos metodológicos quantitativos e descritivos, por meio de questionário estruturado em escala survey online para coleta de dados com estudantes de graduação e pós-graduação. Como técnicas estatísticas de avaliação, foram realizadas análise fatorial e modelagem de equações estruturais.

Este estudo contribui para a discussão de como fatores de recompensa organizacionais influenciam na satisfação de colaboradores e, consequentemente, para melhoria nos resultados e no desempenho. $\mathrm{O}$ estudo possibilita ainda avançar empiricamente na busca de fatores que permitem compreender a percepção da Geração Z sobre o mercado de trabalho.

O trabalho encontra-se dividido em cinco seções. Inicia com esta introdução; a segunda seção aborda a revisão teórica e a construção das hipóteses acerca da relação entre remuneração estratégica, justiça distributiva, motivação e satisfação; a terceira seção descreve a metodologia adotada, os constructos da pesquisa, apresenta a população e a amostra e descreve os procedimentos de coleta e análise dos dados; a quarta seção apresenta os resultados, análises e discussões, e; por fim, na quinta seção são apresentadas as considerações finais, limitações e sugestões para novas pesquisas. 


\section{REVISÃO TEÓRICA E HIPÓTESES DA PESQUISA}

\subsection{Justiça distributiva e remuneração estratégica}

A justiça distributiva evoluiu a partir dos preceitos da teoria da equidade proposta por Adams (COLQUITT et al., 2001). Embora poucas alterações tenham sido feitas, os conceitos mais utilizados na literatura caracterizam-na como a maneira com que a remuneração financeira, promoções e sanções são equitativamente proporcionadas pelas organizações aos colaboradores (REGO, 2002). Gomide Junior e Siqueira (2008) conceituam-na como a distribuição de bens escassos.

Estudos contemporâneos tratam a justiça distributiva na perspectiva de como as organizações propõem a remuneração estratégica. Andrade (2010) investigou a existência de diferenças significativas entre os resultados variáveis e confirma a proposição de que trata-se de um componente essencial para avaliação do clima organizacional; Odelius e Santos (2008) avaliaram e identificaram significativas diferenças de percepção de justiça organizacional entre empregados que recebem remuneração fixa e os que, além desta, percebem remuneração variável (estratégica).

Ahmad e Abdolkhalegh (2015) examinaram a relação dos aspectos da justiça organizacional na satisfação profissional e motivação de funcionários bancários do banco Refah em Kohgiluyeh e Byer-Ahmad e os resultados mostraram que as dimensões mais importantes da justiça organizacional na previsão e explicação da satisfação no trabalho são a justiça distributiva e a justiça interacional e que a justiça distributiva é o único fator de três fatores que, nos resultados dos efeitos de correlação e regressão, tanto na motivação do trabalho quanto nas variáveis de satisfação no trabalho.

A remuneração estratégica desempenha um papel crucial na oferta de mão-de-obra em organizações. Lazear (1979) afirma que o uso dessa forma de retorno é essencial para garantir que os trabalhadores continuem a atuar com efetividade no trabalho. Lazear (1990) destaca ainda que este modelo de remuneração contribui para que os trabalhadores exerçam suas funções com maior afinco e também afeta seu desejo de permanecer no cargo. De acordo com o autor, as taxas de rotatividade em geral são fundamentalmente influenciadas por formatos inadequados de compensação.

Entretanto, a literatura acerca das características de remuneração, concentradas em níveis organizacionais, cresceu lentamente até meados de 1985 e a partir daí diversos estudos passaram a avaliar estruturas e formatos diferenciados de remuneração (Murphy, 1999). Prendergast (1999) abrangeu aspectos remuneratórios orientados à economia; Pavlik, Scott e Tiessen (1993) analisaram modelos de remuneração utilizando literatura financeira e contábil; Indjejikian (1999) avaliou formas de compensação baseadas na contabilidade; Merchant, Van der Stede e Zheng (2003) compararam e contrastaram a literatura contábil orientada à remuneração em diferentes orientações disciplinares; Oyadomari et al. (2009) identificaram práticas de remuneração estratégicas em organizações para associar o alcance das metas com o efeito motivador na busca por desempenho.

Novos estudos direcionaram esforços para fatores estratégicos, de competitividade e relacionados ao desempenho. Nascimento, Franco e Cherubin (2012) avaliaram a remuneração estratégica comparativamente com os indicadores econômico-financeiros para identificar fatores positivos. Larkin, Pierce e Gino (2012) construíram um quadro teórico a fim de examinar como os custos psicológicos e o excesso de confiança reduzem a eficácia da remuneração estratégica baseada em desempenho para avaliar como os profissionais são atraídos e retidos a partir da remuneração estratégica. Nichele, Stefano e Raifur (2015) identificaram fatores estimuladores de atração de talentos e de estímulo de permanência na organização. Ceribelli et al. (2015) descreveram práticas de remuneração estratégica adotadas pelas organizações.

Mais recentemente, Dal Vesco, Beuren e Popik (2016), estudaram a relação entre justiça processual e satisfação não foi significante, já para o grupo de hipóteses que relaciona satisfação no trabalho com avaliação de desempenho, "a relação entre as variáveis latentes confirma a hipótese de que as percepções de justiça na avaliação de desempenho estão positivamente relacionadas com a satisfação no trabalho, condizente com os resultados de Colquitt (2001), 
Sotomayor (2007) e Santos (2010)" (DAL VESCO; BEUREN; POPIK, 2016, p. 134).

Assim, a emergente busca em proporcionar condições favoráveis aos colaboradores para manterem-se nas organizações, associada com a inserção dos preceitos de justiça distributiva como forma de equalizar os retornos de acordo com a produtividade e desempenho dos colaboradores, sugere a avaliação da seguinte hipótese de pesquisa:

Para indivíduos da Geração Z,

- Hipótese 1: a justiça distributiva está positivamente relacionada à remuneração estratégica nas organizações.

\subsection{Justiça distributiva e motivação}

Reter profissionais que agreguem às empresas vem sendo uma apreensão imutável das organizações, especialmente no que tange os jovens da Geração Z que também estão sendo introduzidos no mercado de trabalho e apresentam algumas características distintas das demais gerações. Neste contexto, organizações estão apreensivas em como receber esses profissionais e como sua chegada impactará no clima e motivação organizacional.

A Geração Z tem como componente principal a ambientação com a tecnologia, que já fez parte do seu cotidiano durante toda sua trajetória. Essa familiaridade é algo natural e faz com que o universo digital, a velocidade das informações e a globalização sejam oportunidades para os negócios das empresas. Também há um contraponto a ser observado no que tange às questões de motivação e satisfação desses indivíduos, pois muitas vezes esses profissionais não tem o foco, como seu ponto forte e poderão tornar-se profissionais dispersos, que se concentram pouco em uma só ocupação. Um segundo cuidado para empresas tradicionais é que esse perfil profissional deseja desafios constantes, e reter esses talentos se torna um desafio (VEIGA NETO et al., 2015)

Ao mesmo tempo, a aceleração da competitividade especialmente pela globalização dos mercados e pela tecnologia que constantemente oferece novas possibilidades, requer profissionais com este perfil da Geração Z. Porém, a rotatividade do corpo fun- cional de organizações se torna preocupante pois não querem investir em talentos que possam sair da corporação. Existe todo um esforço no recrutamento e seleção de pessoal especializado, mas isso é insuficiente. Então, criar programas de incentivo à permanência, motivação e satisfação se torna uma estratégia crucial para as firmas.

Apesar da motivação ser algo intrínseco e imperativo ao ser humano, criar oportunidades para ela despontar se tornou recorrente. Neste contexto, a corrente teórica da motivação aqui adotada é a da Hierarquia das Necessidades Humanas estudada por Maslow (1954) e Herzberg (1964), especialmente no que tange os dois fatores motivacionais de Herzberg chamados de higiênicos e motivacionais. Os fatores higiênicos estão pautados na qualidade do espaço de trabalho, salário, benefícios, entre outros. Já fatores motivacionais são pautados a partir do crescimento, sendo que a incumbência de responsabilidades pode auxiliar na evolução das tarefas da equipe e promover a motivação, inclusive coletiva (NOVAES, 2007, p. 09).

"Os fatores motivacionais são intrínsecos ao trabalho e derivam do conteúdo do cargo e do seu nível de enriquecimento, diferentemente dos fatores higiênicos que são periféricos ao trabalho" (HERZBERG, 1986, p. 12). Herzberg (1986) relata que, somente os "sistemas de necessidades que correspondem aos níveis de estima e auto realização de Maslow (1970), convêm de fontes diretas de motivação para se trabalhar com eficiência".

Neste sentido, Maslow (1970, p. 05) estabeleceu a Hierarquia das Necessidades Humanas quais são classificadas na ordem: 1) fisiológicas (básicas); 2) segurança; 3) sociais 4) estima; 5) ? e 6) de autorrealização onde essas necessidades são dispostas hierarquicamente, ou seja, somente após as necessidades primárias serem saciadas, o ser humano procura a satisfação das demais.

Maslow (1954) e Herzberg (1964) concordam em relação a ordem das necessidades especialmente no que tange os fatores motivacionais e higiênicos as necessidades de autorrealização e básicas. Observa-se que a condição de motivação pode ser diferente em indivíduos ou de acordo com a experiência vivenciada num determinado momento. Em virtude disso, o estado de total satisfação nem sempre é alcançado ou às vezes ocorre por um determinado período. Então, 
criar estratégias para tornar a motivação permanente é um desafio mas, em contrapartida, quanto maior o engajamento, mais resultados. Evidentemente, a justiça distributiva surge neste contexto pois se refere à maneira como a organização distribui seus recursos entre os seus membros, incluindo salários, pontuações nas avaliações de desempenho, ações disciplinares ou repartição de recursos orçamentários entre as unidades (Palozolli, 2000).

A justiça distributiva relaciona-se com a forma como os indivíduos avaliam as distribuições de bens, que podem ser positivos, quando caracterizados como renda, liberdade ou cargos políticos ou, negativos quando nela estão inseridas punições, sanções ou penalidades (SAMPAIO et al., 2009). Também, tem relação com o sentimento de equidade, como por exemplo, quando a distribuição de resultados é percebida como justa, ou seja a razão entre inputs (investimentos e contribuições) e outcomes (resultados) é igual à razão entre investimentos e resultados, se comparado a outra pessoa; se houver desigualdade, pode ocasionar tensões (ASSMAR et al., 2005).

$\mathrm{Na}$ abordagem unidimensional da justiça distributiva há princípios distributivos únicos e que são aplicados em todas as situações de distribuição e, na abordagem multidimensional, são diversos princípios que orientam os julgamentos, que podem ser utilitaristas, equitativos ou benevolentes, de acordo com as particularidades que exigirem cada situação (SAMPAIO et al., 2009). Dessa forma, as teorias relacionadas à motivação estão ligadas à percepção da justiça distributiva, pois os membros da organização esperam que seus esforços sejam compensados de maneira justa, perante aos demais. Caso o indivíduo venha a perceber desequilíbrio nessas distribuições, isso poderá refletir sensivelmente nos aspectos motivacionais, incorrendo em prejuízos tanto individuais como para a organização. Diante desse contexto, são apresentadas as hipóteses 2 e 3 desta pesquisa:

Para indivíduos da Geração Z,

- Hipótese 2: a percepção da justiça distributiva afeta positivamente a motivação.

- Hipótese 3: a percepção da justiça distributiva, por meio da remuneração estratégica, propicia motivação.

\subsection{Justiça Distributiva e Satisfação no Trabalho}

A justiça distributiva leva à satisfação com os resultados e consequentemente leva o indivíduo a cooperar (REGO, 2002; KIM; MAUBORGNE, 1997). Para Palozolli (2000), a justiça distributiva se relacionada à maneira como a organização distribui os recursos entre os seus membros, incluindo salários, pontuações nas avaliações de desempenho, ações disciplinares ou repartição de recursos orçamentários entre as unidades.

Com base nas teorias motivacionais de Herzberg (1986) e de Maslow (1970), a percepção da justiça distributiva poderia impactar na motivação e satisfação. As características ligadas à tecnologia e informação os coloca em redes de contatos diversas e a Geração Z pode tender a ser mais aberta a partilhar e conseguir informações, ao mesmo tempo que tende a ser individualista, com grande dificuldade em realizar trabalhos em equipe e em compreender a si mesma. As três palavras que a definem velocidade, conexão e interatividade (VEIGA NETO et al., 2015).

A justiça organizacional tem forte impacto nas relações entre trabalhadores e organizações e a relação entre justiça e equidade influenciam nas atitudes e comportamentos dentro desse ambiente (ASSMAR et al., 2005). Por essa razão, os gestores ao conhecerem como esses reflexos prejudicam a organização, podem tomar decisões que podem prevenir conflitos (ASSMAR et al., 2005). Esses conflitos podem gerar desconforto e insatisfação. Tadeucci (2009) reforça a afirmação de Spector (2006) de que a insatisfação com o trabalho provoca rotatividade, gera altos custos operacionais e incorre em perda do conhecimento que já foi gerado. As recompensas positivas são mais prováveis de gerar satisfação do que punições e castigos, uma vez que essas últimas podem trazer sentimentos negativos que podem induzi-los a cometer pequenas faltas, sejam intencionais ou não, além de favorecer um clima desagradável de trabalho (TADEUCCI, 2009).

Mas o que gera satisfação nos indivíduos e como ela está relacionada com a justiça distributiva? A satisfação no trabalho está mais ligada à percepção da avaliação de desempenho, da distribuição de salários, lucros e de como os gestores tomam decisões e 
interagem com os indivíduos afetados, do que com a forma como os processos são executados. Com isso, a percepção das três dimensões de justiça organizacional na avaliação de desempenho pode melhorar a satisfação no trabalho (DAL VESCO, BEUREN; POPIK, 2016). A Figura 1 apresenta algumas abordagens relacionadas à percepção de outros autores sobre a satisfação relacionado ao ambiente de trabalho.

Figura 1 Percepções sobre satisfação relacionada ao ambiente de trabalho

\begin{tabular}{|c|c|}
\hline Percepções sobre satisfação & Autores \\
\hline $\begin{array}{l}\text { - O controle sobre dada tarefa proporciona maior satisfação } \\
\text { - Influência na saúde, qualidade de vida e comportamento } \\
\text { - As empresas devem possibilitar o aumento do bem-estar e satisfação de seus empregados. }\end{array}$ & $\begin{array}{l}\text { Dawal, Taha e Ismail (2009) } \\
\text { Martinez e Paraguay (2003) } \\
\text { Bowling (2007) }\end{array}$ \\
\hline $\begin{array}{l}\text { - Promove à rentabilidade, à produtividade, à retenção de empregados e clientes satisfeitos. } \\
\text { - Está relacionada aos fatores da organização no trabalho. } \\
\text { - Empregados satisfeitos proporcionam maior satisfação aos clientes e isso influencia de forma positi- } \\
\text { va o desempenho da organização. } \\
\text { - As pessoas desempenham melhor seu trabalho quando estão satisfeitas. }\end{array}$ & $\begin{array}{l}\text { Palaiologos e Papazekos; } \\
\text { Panayotopoulou (2011) } \\
\text { Dawal, Taha e Ismail (2009) } \\
\text { Papazekos e Panayotopoulou (2011) }\end{array}$ \\
\hline $\begin{array}{l}\text { E equidade percebida no pagamento tem um efeito direto na satisfação. } \\
\text { - A satisfação no trabalho resulta diretamente das percepções de justiça processual. } \\
\text { - A satisfação dos empregados com os resultados salariais decorre tanto das percepções de justiça } \\
\text { distributiva quanto das percepções de justiça processual. }\end{array}$ & $\begin{array}{l}\text { Cavanagh (1992) } \\
\text { Summers e Hendrix (1991) } \\
\text { Alexander e Ruderman (1987) } \\
\text { Folger e Konovski (1989) }\end{array}$ \\
\hline
\end{tabular}

Fonte extraído e adaptado de Dal Vesco et al. (2016).

De acordo com a percepção dos autores, as fontes de satisfação dos indivíduos nos ambientes organizacionais estão relacionadas aos fatores propostos por Herberg (1986), sendo os higiênicos (incluindo a organização do trabalho, controle sobre as tarefas, qualidade de vida e bem-estar), e os fatores motivacionais (dentre eles, a equidade e a justiça). Diante da abordagem da justiça distributiva e da satisfação no trabalho, são apresentadas as hipóteses 4, 5 e 6 desta pesquisa: para indivíduos da Geração Z,

- Hipótese 4: a percepção da justiça distributiva relaciona-se positivamente com a satisfação no trabalho.

- Hipótese 5: a percepção da justiça distributiva, por meio da remuneração estratégica, propicia satisfação no trabalho.

- Hipótese 6: a motivação propicia satisfação no trabalho.

\section{PROCEDIMENTOS METODOLÓGICOS}

O esboço descritivo da pesquisa seguiu os preceitos da teoria de justiça distributiva e sua relação com a remuneração estratégica e o nível de satisfação e motivação para o trabalho de indivíduos da Geração $Z$, estudantes universitários do Oeste e Meio Oeste do Estado de Santa Catarina, Brasil. A opção por esse universo ocorreu pela disponibilidade de participação da pesquisa e, especialmente, pelo interesse dos autores em compreender a relação proposta nos objetivos.

O problema de pesquisa preconizou a uma pesquisa quantitativa, por meio de técnicas de estatística multivariada que envolveu análise fatorial e regressão. O universo da pesquisa abrangeu estudantes universitários da graduação e da pós graduação de uma instituição comunitária de ensino de Santa Catarina, com cerca de 13 mil estudantes. A amostra resultou em 1.258 estudantes, número superior ao que o cálculo estatístico para composição da amostra indicou, 856 indivíduos, considerando um erro amostral de 1\%. Desse montante, foram exclusas respostas inválidas ou incompletas e também que não se enquadraram no perfil da Geração Z. A amostra final válida de 1.112 respondentes. 
Utilizou-se um questionário estruturado com perguntas fechadas, dividido em cinco blocos distintos (Apêndice A). Um bloco com dados relativos a identificação dos respondentes e outros quatro blocos elaborados com base nos construtos criados a partir das hipóteses. O questionário foi elaborado com escalas métricas para reconhecer o perfil dos participantes e escalonamento nominal/categórico entre 1 e 7 para reconhecer a ausência ou presença do atributo, onde dummy zero e um indica não ter significado quantitativo, ou seja, foi usado para identificar a ausência ou presença do atributo. As escalas métricas utilizadas são intervalares modelo Likert de 7 pontos (HAIR JR et al., 2005).

Figura 2 Modelo teórico

\begin{tabular}{|c|c|c|}
\hline Hipóteses & 口 Dimensão/Construção & Autores (ano) \\
\hline $\begin{array}{l}\text { H1: A justiça distributiva } \\
\text { está positivamente relacio- } \\
\text { nada à remuneração estra- } \\
\text { tégica nas organizações. }\end{array}$ & $\begin{array}{l}\text { - A percepção da justiça distributiva reflete na motivação dos membros da } \\
\text { organização, ao sentirem se esta distribui seus recursos (salários, premiações, } \\
\text { punições, etc.) de maneira justa e igualitária. } \\
\text { - Profissionais levam muito em consideração o salário em suas decisões, mas } \\
\text { o grande desafio das empresas está em definir qual a remuneração é mais } \\
\text { adequada seguindo a filosofia da organização e a que melhor se enquadra } \\
\text { em seu quadro funcional, visto ser um paradigma entre os dois interessados. } \\
\text { - Existem diferenças significativas entre os resultados variáveis que indicam a }\end{array}$ & $\begin{array}{l}\text { Lazear (1990); Palozolli } \\
\text { (2000); Colquitt (2001), } \\
\text { Sotomayor (2007) e Santos } \\
\text { (2010). Assmar et al. (2005); } \\
\text { Sampaio et al. (2009); } \\
\text { Tadeucci (2009); Trindad et } \\
\text { al. (2011); Andrade (2010); } \\
\text { Dalvesco et al. (2016). }\end{array}$ \\
\hline
\end{tabular}
percepção de justiça distributiva [...] trata-se de um componente essencial para avaliação do clima organizacional.

- O modelo de remuneração estratégica contribui para que os trabalhadores exerçam suas funções com maior afinco e também afeta seu desejo de permanecer no cargo.

- A hipótese de que as percepções de justiça na avaliação de desempenho estão positivamente relacionadas com a satisfação no trabalho, condizente com os resultados de

\section{H2: a percepção da justiça distributiva afeta positiva- mente a motivação. \\ H3: a percepção da justiça distributiva, por meio da remuneração estratégica, propicia motivação.}

- Fatores motivacionais (crescimento, sendo que a incumbência de responsabilidades pode auxiliar na evolução das tarefas da equipe e promover a motivação, inclusive coletiva) são intrínsecos ao trabalho e derivam do conteúdo do cargo e do seu nível de enriquecimento, diferentemente dos fatores higiênicos (qualidade do espaço de trabalho, salário, benefícios, entre outros) que são periféricos ao trabalho.

- A justiça distributiva relaciona-se com a forma como os indivíduos avaliam as distribuições de bens, que podem ser positivos, quando caracterizados como renda, liberdade ou cargos políticos ou, negativos quando nela estão inseridas punições, sanções ou penalidades.

- Quando a distribuição de resultados é percebida como justa, ou seja a razão entre inputs (investimentos e contribuições) e outcomes (resultados) é igual à razão entre investimentos e resultados, se comparado a outra pessoa; se houver desigualdade, pode ocasionar tensões.

H4: a percepção da justiça distributiva relaciona-se positivamente com a satisfação no trabalho.

H5: a percepção da justiça distributiva, por meio da remuneração estratégica, propicia satisfação.

H6: a motivação propicia satisfação no trabalho.
- A justiça distributiva leva à satisfação com os resultados e consequentemente leva o indivíduo a cooperar.

- A justiça organizacional tem forte impacto nas relações entre trabalhadores e organizações e a relação entre justiça e equidade influenciam nas atitudes e comportamentos dentro desse ambiente.

- O controle sobre dada tarefa proporciona maior satisfação

- Influência na saúde, qualidade de vida e comportamento está relacionada aos fatores da organização no trabalho.

- As pessoas desempenham melhor seu trabalho quando estão satisfeitas.

- A satisfação no trabalho resulta diretamente das percepções de justiça processual.

- A satisfação dos empregados com os resultados salariais decorre tanto das percepções de justiça distributiva quanto das percepções de justiça processual.
Herzberg (1986); Sampaio et al. (2009); Assmar et al. (2005).
Rego (2002); Kim; Mau borgne (1997); Assmar et al. (2005):

Dawal, Taha e Ismail, (2009),

Palaiologos, Papazekos e

Panayotopoulou (2011);

Folger e Konovski, (1989),

Dal Vesco, Beuren e Popik

(2016).

Fonte Os autores (2018). 
Após construção do questionário, optou-se por validá-lo com pesquisador da área. Além disso, realizou-se um pré-teste com 2 estudantes a fim de evitar possíveis falhas entre o contexto teórico e a compreensão dos respondentes. Assim, na Figura 2 apresentam-se os relacionamentos sugeridos entre os construtos, bem como os autores basilares utilizados para amparar a aplicação empírica do modelo.

A Figura 2 apresenta detalhadamente a construção das hipóteses, bem como os autores que serviram de base. Com base nisso, foi possível elaborar o diagrama constante na Figura 3. O diagrama demonstra a relação existente entre as variáveis do constructo, e de que forma testou-se, empiricamente, o modelo proposto.

Figura 3 Diagrama do modelo teórico e hipóteses de pesquisa.

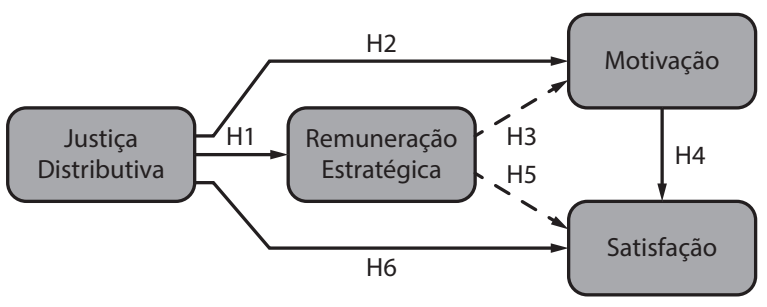

Fonte Os autores (2018)

As variáveis componentes do modelo estrutural, indicam comparação e medição dos constructos. Para análise dos dados, fora validado mediante análise exploratória, análise fatorial confirmatória e modelagem de equações estruturais (SEM) com utilização do software AMOS (SPSS).

\section{RESULTADOS, ANÁLISE E DISCUSSÕES}

\subsection{Descrição dos respondentes}

Esta seção apresenta as informações resultantes da pesquisa realizada com 1.112 estudantes que fazem parte da Geração Z, que são os indivíduos nascidos a partir de 1989 e que em 2018 estão na faixa dos 17 aos 29 anos.

Diante do universo pesquisado, na Tabela 6 é apresentada a descrição da amostra, com as análises socioeconômicas e demográficas dos estudantes que participaram da pesquisa.
A média das idades ficou em 21 anos, com um desvio padrão de 3,12 e variância estatística de 9,78. Os dados demonstram que a maioria deles são do sexo feminino $(70,7 \%)$, sendo que $78 \%$ tem até 23 anos. Dentre os pesquisados, $71 \%$ estão trabalhando ou fazendo estágio, 17,4\% já trabalharam, mas estão desempregados e $11,8 \%$ nunca trabalharam.

Quanto à área dos estudantes que estão atuando no mercado de trabalho $16,7 \%$ estão em áreas administrativas; $9,9 \%$ atuam como atendentes/secretários/ recepcionistas. Dos $8,6 \%$ que estão realizando estágio, $4,1 \%$ não especificaram a área e os demais atuam na contabilidade, engenharia, saúde, jurídica, indústrias etc. No setor comercial atuam $8,4 \%$, na contabilidade $7,6 \%$, serviços públicos $5 \%$, área jurídica $4,5 \%$, serviços gerais $4,5 \%$, saúde $4 \%$, TI $4 \%$, indústria $3,8 \%$, educação 3,3\%, marketing/publicidade/propaganda $2,7 \%$, agricultura $2,5 \%$, engenharia e arquitetura $2,5 \%$, recurso humanos $2 \%$ e outras áreas diversas $8,4 \%$.

Dentre os $71 \%$ que estão trabalhando ou fazendo estágio, as remunerações recebidas variam de até 1 salário mínimo para $36,5 \%$; entre 1 a 3 para 59,6\%; entre 3 a 5 para $3 \%$ e apenas $0,9 \%$ recebem mais que 5 salários mínimos. A maioria dos pesquisados (59,5\%) atua na mesma área que estuda ou em áreas afins.

\subsection{Análise exploratória dos dados e modelagem}

A Tabela 2 apresenta os constructos denominados como Justiça Distributiva (JD), Remuneração Estratégica (RE), Motivação (M) e Satisfação (S).

O constructo "Justiça Distributiva" foi relacionado neste estudo com três blocos e dezoito assertivas, sendo três delas reversas (Busca emprego: currículo; Busca emprego: vagas poucos interessados, Empresa Ideal: esforço) para testar a atenção dos respondentes, sendo que essas deram abaixo do ideal, confirmando a confiança dos dados.

Dessa forma, os resultados, de acordo com a Tabela 7, indicam médias significativas, todas acima de 4 pontos, com indicativos de que os indivíduos estão atentos ao que o mercado de trabalho oferece. Quanto a empresa ideal para trabalhar, os resultados mostram que para a Geração Z, seguem os princípios da justiça distributiva e da remuneração estratégica (Lazear (1990); Trindad, et al. (2011)). 
Tabela 1 Informações socioeconômicas e demográficas dos participantes da pesquisa (em \%)

\begin{tabular}{|c|c|c|c|}
\hline \multicolumn{4}{|c|}{ Sexo } \\
\hline Masculino & 29,3 & Feminino & 70,7 \\
\hline \multicolumn{4}{|c|}{ Idade } \\
\hline Até 20 anos & 48,3 & De 24 a 26 anos & 14,2 \\
\hline De 21 a 23 anos & 29,7 & De 27 a 29 anos & 7,8 \\
\hline \multicolumn{4}{|c|}{ Situação atual de trabalho } \\
\hline Nunca trabalhou & 11,6 & Trabalha mas está a procura de um novo emprego & 27,3 \\
\hline Já trabalhou mas está desempregado & 17,4 & Faz estágio & 13,8 \\
\hline Não trabalha e não estou buscando uma oportunidade & 0 & Trabalha há menos de 1 ano & 4,4 \\
\hline Trabalha e não está buscando uma oportunidade & 5,3 & Trabalha há mais de 1 ano & 20,2 \\
\hline \multicolumn{4}{|c|}{ Área de atuação } \\
\hline Administrativa & 16,7 & Saúde & 4,0 \\
\hline Atendente/Secretaria/Recepção & 9,9 & Tecnologia de Informação & 4,0 \\
\hline Estágio em áreas diversas & 8,6 & Indústria & 3,8 \\
\hline Comercial & 8,4 & Educação & 3,3 \\
\hline Contabilidade & 7,6 & Marketing & 2,7 \\
\hline Serviço público & 5,0 & Agricultura & 2,5 \\
\hline Área jurídica & 4,5 & Engenharia/arquitetura & 2,5 \\
\hline Serviços gerais & 4,5 & Recursos humanos & 2,0 \\
\hline Outro & 8,4 & & \\
\hline \multicolumn{4}{|c|}{ Nível salarial dos que trabalham } \\
\hline Até 1 salário mínimo & 36,5 & Entre 3 e 5 salários mínimos & 3 \\
\hline Entre 1 e 3 salários mínimos & 59,6 & Mais que 5 salários mínimos & 0,9 \\
\hline \multicolumn{4}{|c|}{ A área que atua é a mesma em que estuda? } \\
\hline $\operatorname{sim}$ & 44 & Não & 40,5 \\
\hline Área afins & 15,5 & & \\
\hline
\end{tabular}

Fonte Dados da pesquisa (2018).

O constructo "Remuneração Estratégica" [RE] abarcou dois blocos com vinte e uma assertivas, que também atingiu média acima de 4 pontos, sugerindo que os diferentes tipos de remuneração estratégica são importantes para essa geração, o que corrobora com Trindad et al. (2011) de que os profissionais levam muito em consideração o salário em suas decisões e também o estudo de Lazear (1990) quando afirma que o modelo de remuneração estratégica contribui para que os trabalhadores exerçam suas funções com maior afinco sendo que isso também afeta o desejo de permanecer no cargo.

O terceiro constructo "Motivação" $[\mathrm{M}]$, reflete um bloco com dezesseis assertivas sendo uma delas reversa. Os resultados sugerem que a motivação está ligada aos aspectos de reconhecimento (6,46 pontos), ou seja, para a Geração Z o reconhecimento é um fator relevante para se sentir motivado no trabalho. A hipótese de Dal Vesco et al. (2016) é que esse controle proporciona maior satisfação, o que se confirma na pesquisa. Outro fator a se destacar é que a Geração $Z$ 
Tabela 2 Variáveis e descrição dos dados da pesquisa

\begin{tabular}{|c|c|c|c|c|c|c|c|c|}
\hline \multirow{2}{*}{ ID } & \multirow{2}{*}{ Variáveis } & \multirow{2}{*}{ Média } & \multirow{2}{*}{$\begin{array}{l}\text { Desvio } \\
\text { padrão }\end{array}$} & \multirow{2}{*}{ Variação } & \multicolumn{2}{|c|}{ Assimetria } & \multicolumn{2}{|c|}{ Curtose } \\
\hline & & & & & Estat. & Erro pad. & Estat & Erro pad. \\
\hline \multirow{4}{*}{ JD } & Recompensa justa & 6,16 & 1,255 & 1,575 & $-1,808$ &, 073 & 3,287 &, 147 \\
\hline & Recompensa contribui & 5,99 & 1,320 & 1,743 & $-1,669$ &, 073 & 2,856 &, 147 \\
\hline & Recompensa experiência & 4,73 & 1,803 & 3,249 &,- 532 &, 073 &,- 617 &, 147 \\
\hline & Recompensa demais salários & 4,35 & 1,871 & 3,501 &,- 301 &, 073 &,- 942 &, 147 \\
\hline \multirow{21}{*}{ RE } & Acionaria & 4,51 & 1,718 & 2,950 &,- 367 &, 073 &,- 589 &, 147 \\
\hline & Funcional & 5,38 & 1,525 & 2,325 &,- 951 &, 073 &, 389 &, 147 \\
\hline & Habilidades & 5,57 & 1,509 & 2,277 & $-1,200$ &, 073 & 1,047 &, 147 \\
\hline & Benefícios & 5,78 & 1,433 & 2,054 & $-1,464$ &, 073 & 1,985 &, 147 \\
\hline & Lucros Resultados & 5,69 & 1,526 & 2,327 & $-1,337$ &, 073 & 1,348 &, 147 \\
\hline & Distribuição Ganhos & 5,26 & 1,632 & 2,664 &,- 961 &, 073 &, 335 & ,147 \\
\hline & Bonificação & 5,67 & 1,525 & 2,327 & $-1,306$ &, 073 & 1,211 &, 147 \\
\hline & Benefícios Sociais & 5,49 & 1,671 & 2,792 & $-1,147$ &, 073 & 605 &, 147 \\
\hline & Assistência Financeira & 5,45 & 1,592 & 2,533 &,- 962 &, 073 &, 298 &, 147 \\
\hline & Auxílio Médico / Odontológico & 6,10 & 1,366 & 1,867 & $-1,802$ &, 073 & 3,014 &, 147 \\
\hline & Auxílio Creche & 5,14 & 1,886 & 3,557 &,- 767 &, 073 &,- 539 &, 147 \\
\hline & Auxílio Educação & 5,95 & 1,499 & 2,248 & $-1,598$ &, 073 & 1,969 &, 147 \\
\hline & Esporte, cultura, lazer & 5,22 & 1,698 & 2,885 &,- 783 &, 073 &,- 250 &, 147 \\
\hline & Flexibilidade de horário & 5,80 & 1,484 & 2,202 & $-1,339$ &, 073 & 1,189 &, 147 \\
\hline & Home-office & 5,07 & 1,688 & 2,850 &,- 682 &, 073 &,- 328 &, 147 \\
\hline & Previdência Privada & 5,36 & 1,623 & 2,633 &,- 927 &, 073 & 187 &, 147 \\
\hline & Refeitório & 5,67 & 1,564 & 2,446 & $-1,166$ &, 073 &, 656 &, 147 \\
\hline & Seguro Vida & 5,75 & 1,610 & 2,593 & $-1,337$ &, 073 & 1,068 &, 147 \\
\hline & Telefone celular & 4,13 & 2,010 & 4,038 &,- 149 &, 073 & $-1,171$ &, 147 \\
\hline & Ticket alimentação & 5,88 & 1,533 & 2,349 & $-1,499$ &, 073 & 1,678 &, 147 \\
\hline & Transporte diferenciado & 4,66 & 1,955 & 3,823 &,- 464 &, 073 &,- 909 &, 147 \\
\hline \multirow{16}{*}{ M } & Decisões org. & 5,27 & 1,507 & 2,272 &,- 782 &, 073 &, 143 & ,147 \\
\hline & Confio & 5,58 & 1,548 & 2,396 & $-1,121$ &, 073 &, 708 &, 147 \\
\hline & Me desenvolvo profissionalmente & 6,28 & 1,212 & 1,468 & $-2,254$ & 073 & 5,544 &, 147 \\
\hline & Me relaciono chefia & 5,18 & 1,717 & 2,947 &,- 863 &, 073 &,- 046 &, 147 \\
\hline & Me relaciono equipe & 5,99 & 1,441 & 2,076 & $-1,781$ &, 073 & 2,904 &, 147 \\
\hline & Status & 4,35 & 1,967 & 3,868 &,- 306 &, 073 & $-1,031$ &, 147 \\
\hline & Retenção talentos & 4,91 & 1,813 & 3,288 &,- 634 &, 073 &,- 538 &, 147 \\
\hline & Salário atração & 4,43 & 1,842 & 3,394 &,- 377 &, 073 &,- 829 &, 147 \\
\hline & Autonomia & 5,57 & 1,537 & 2,362 & $-1,195$ &, 073 &, 928 &, 147 \\
\hline & Realização pessoal & 6,43 & 1,163 & 1,354 & $-2,564$ &, 073 & 6,907 &, 147 \\
\hline & Ideias ouvidas & 6,33 & 1,210 & 1,464 & $-2,384$ &, 073 & 6,106 &, 147 \\
\hline & Reconhecido Trabalho Executo & 6,46 & 1,148 & 1,318 & $-2,794$ &, 073 & 8,255 &, 147 \\
\hline & Reconhecimento não é importante & 1,97 & 1,758 & 3,089 & 1,809 &, 073 & 2,008 &, 147 \\
\hline & Comunicação Informações & 5,76 & 1,445 & 2,087 & $-1,262$ &, 073 & 1,167 &, 147 \\
\hline & Benefício melhor salario & 4,28 & 1,806 & 3,260 &,- 211 &, 073 &,- 850 &, 147 \\
\hline & Sentir atraído & 5,13 & 2,008 & 4,032 &,- 866 &, 073 &,- 501 &, 147 \\
\hline \multirow{8}{*}{$\mathbf{S}$} & Reconhecimento & 6,46 & 1,179 & 1,390 & $-2,903$ &, 073 & 8,909 &, 147 \\
\hline & Benefícios & 5,84 & 1,424 & 2,027 & $-1,451$ &, 073 & 1,890 &, 147 \\
\hline & Comunicação & 6,11 & 1,269 & 1,612 & $-1,863$ &, 073 & 3,651 &, 147 \\
\hline & Integração Colaboradores & 5,97 & 1,360 & 1,849 & $-1,614$ &, 073 & 2,473 &, 147 \\
\hline & Valorização Salarial & 6,22 & 1,215 & 1,477 & $-2,122$ &, 073 & 4,996 &, 147 \\
\hline & Promoção & 5,87 & 1,420 & 2,015 & $-1,502$ &, 073 & 2,002 &, 147 \\
\hline & Justiça Organizacional & 6,09 & 1,290 & 1,664 & $-1,762$ &, 073 & 3,151 & ,147 \\
\hline & Rotinas & 6,02 & 1,273 & 1,620 & $-1,695$ & ,073 & 3,158 & 147 \\
\hline
\end{tabular}

Fonte dados da pesquisa (2018). 
Satisfação e Motivação no Trabalho: Insights sobre Percepção da

Justiça Distributiva e Remuneração Estratégica por Indivíduos da Geração Z

Tabela 3 Análise Fatorial do Constructo da pesquisa

\begin{tabular}{|c|c|c|c|c|c|c|}
\hline \multirow{2}{*}{ Constructo } & \multirow{2}{*}{ Variáveis } & \multicolumn{4}{|c|}{ Componentes } & \multirow{2}{*}{ Comunalidades } \\
\hline & & 1 & 2 & 3 & 4 & \\
\hline \multirow{5}{*}{ 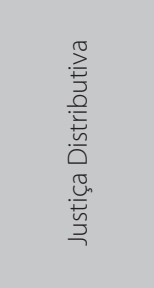 } & Recompensa justa & & & & 0,763 & 0,793 \\
\hline & Recomp_contribui & & & & 0,789 & 0,810 \\
\hline & Recomp_exp & & & & 0,769 & 0,653 \\
\hline & Recomp_demaissalarios & & & & 0,793 & 0,671 \\
\hline & Recomp_justa & & & 0,713 & & 0,665 \\
\hline \multirow{11}{*}{ 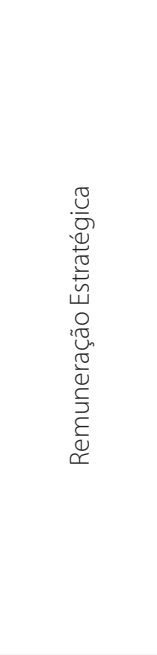 } & LucrosResult & & & 0,795 & & 0,737 \\
\hline & DistrGanhos & & & 0,809 & & 0,779 \\
\hline & Bonif & & & 0,771 & & 0,715 \\
\hline & BenfSociais & & & 0,449 & & 0,370 \\
\hline & B_AssFinanc & & & 0,692 & & 0,665 \\
\hline & B_Méd.Odonto & & & 0,764 & & 0,615 \\
\hline & B_AuxCreche & & & 0,724 & & 0,636 \\
\hline & B_Aux_Educ & & & 0,647 & & 0,507 \\
\hline & B_Refeitorio & & & 0,713 & & 0,618 \\
\hline & B_Seguro_Vida & & & 0,640 & & 0,553 \\
\hline & B_ticketalim & & & 0,713 & & 0,454 \\
\hline \multirow{8}{*}{ 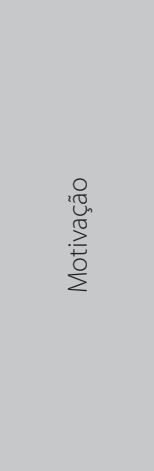 } & Decisoes_orga & 0,578 & & & & 0,547 \\
\hline & Confio & 0,596 & & & & 0,777 \\
\hline & Medesenvolvoprofis & 0,791 & & & & 0,545 \\
\hline & Relacionoequipe & 0,642 & & & & 0,770 \\
\hline & Realizopess & 0,781 & & & & 0,756 \\
\hline & Ideiasouvidas & 0,755 & & & & 0,781 \\
\hline & Reconhecidotrabex & 0,772 & & & & 0,526 \\
\hline & Colnf & 0,638 & & & & 0,645 \\
\hline \multirow{7}{*}{ 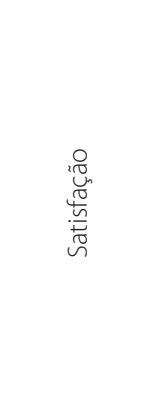 } & Benef & & 0,703 & & & 0,742 \\
\hline & Comun & & 0,711 & & & 0,744 \\
\hline & Integ_Colab & & 0,751 & & & 0,740 \\
\hline & ValorizSalarial & & 0,753 & & & 0,685 \\
\hline & Promo & & 0,764 & & & 0,734 \\
\hline & JustOrg. & & 0,746 & & & 0,671 \\
\hline & Rotinas & & 0,710 & & & 0,793 \\
\hline
\end{tabular}

Fonte dados da pesquisa (2018).

Método de extração Componente principal. Método de rotação: Varimax com normalização de Kaiser. 
busca crescimento profissional ( 6,28 pontos) e pessoal (6,43 pontos). Os dados do constructo $\mathrm{M}$ ainda validam a teoria de Palozolli (2000), Assmar et al. (2005), Sampaio et al. (2009), Tadeucci (2009) e Herzberg (1986) sobre a influência da justiça distributiva na motivação dos membros da organização, ao sentirem se esta distribui seus recursos (salários, premiações, punições, etc.) de maneira justa e igualitária.

O quarto e último constructo "Satisfação" [S] reflete um bloco com oito assertivas sendo que a média indica que os indivíduos da Geração Z, para sentirem-se satisfeitos com o trabalho, valorizam o reconhecimento $(6,46)$, a boa comunicação $(6,11)$, os benefícios $(5,84)$, a valorização salarial $(6,22)$, as rotinas $(6,02)$, a justiça organizacional $(6,09)$ e a integração entre os colaboradores $(5,97)$. Portanto, a literatura de Colquitt (2001), Sotomayor (2007), Santos (2010) e Dalvesco et al. (2016) de que as percepções de justiça na avaliação de desempenho estão positivamente relacionadas com a satisfação no trabalho são validadas neste estudo.

Constatou-se normalidade nos dados, os construtos foram legitimados com a realização da análise fatorial, utilizando-se do método de rotação varimax com normalização de Kaiser. Na Tabela 3 apresentam-se os fatores e os valores os quais encontram-se em normalidade (HAIR et al., 2009).
Para realização da análise fatorial, foram retirados do modelo todos os fatores de análise que apresentaram valores abaixo de 0,455 conforme a literatura sugere (HAIR et al., 2009). Para tanto, o total de 18 variáveis foram eliminadas, permanecendo 4 na dimensão de Justiça Distributiva, 11 na dimensão de Remuneração Estratégica, 8 na dimensão de Motivação e 7 na dimensão Satisfação.

Para fins de aprimoramento do ajuste, no constructo Justiça Distributiva manteve-se apenas o terceiro bloco. No constructo Remuneração Estratégica excluíram-se os indicadores "Remuneração acionária, Funcional (por cargos), Por habilidades, Por competências/benefícios, Espaço cultura, lazer, Flexibilidade de horário, Home office, Previdência complementar, Telefone celular e Transporte diferenciado".

No constructo Motivação excluíram-se os indicadores: "Quando me relaciono com a chefia, Quando meu trabalho me proporciona status, Quando a organização oferece formas de retenção de talentos, Quando fator salarial é o maior foco da empresa para atração de talentos, Quando percebo que tenho autonomia e Ter reconhecimento não é importante" e no constructo Satisfação excluíram-se o indicador "Quando me sentir reconhecido pelo trabalho que realizo" também foi excluído.

Figura 4 Variáveis e descrição dos dados da pesquisa

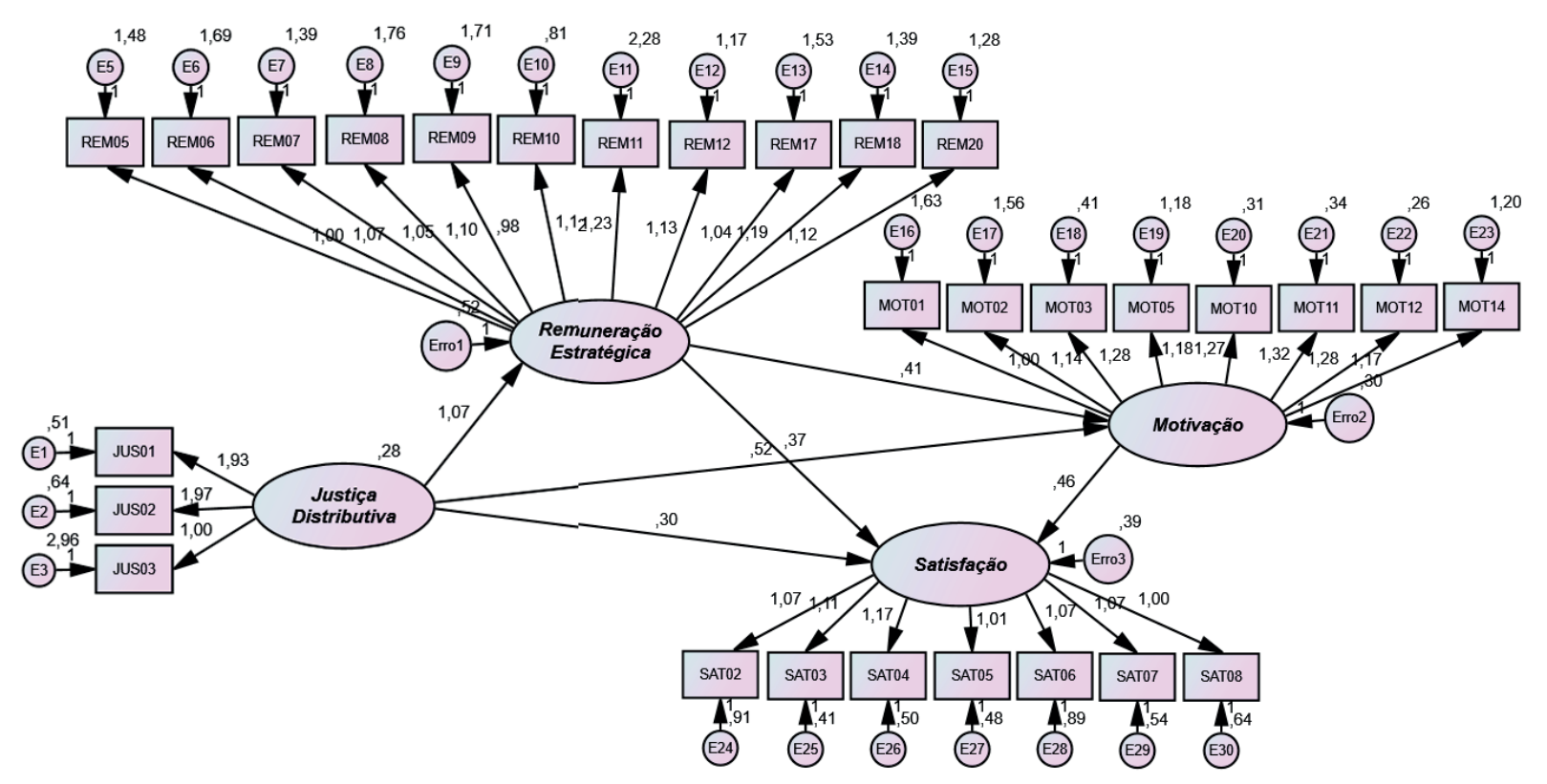

Fonte os autores (2018). 
Realizada a redução das dimensões, foram utilizadas aas variáveis que apresentaram significância para construção do modelo. A modelagem possibilitou construir o desenho estrutural, conforme Figura 4.

A modelagem de equações estruturais iniciou mensurando as cargas e os pesos fatoriais e as significâncias em relação aos indicadores de cada um dos constructos. Em seguida, a análise do modelo estrutural mediu os índices da capacidade preditiva do modelo, bem como os coeficientes estruturais. Para melhoria do ajuste do modelo, a variável "Recomp_demaissalarios", componente da dimensão de Justiça Distributiva foi eliminada, tendo em vista que sua permanência reduzia o poder de explicação do modelo.

Ressalta-se que todos os testes realizados, exibidos na modelagem de equações estruturais apresentaram-se positivos e significantes. Destaca-se que a validade preditiva $\left(R^{2}\right)$ relativa aos valores de referência para a atribuição do grau de explicação preditiva apresentaram-se todos com efeito significativo, ou seja, acima de 26\% (Cohen, 1998). Este resultado pode ainda ser amparado pelos índices de confiabilidade do modelo de mensuração após os ajustes estão apresentados na Tabela 4.
Tabela 4 Análise de confiabilidade dos dados

\begin{tabular}{l|c|c|c|c|c}
\hline \multicolumn{1}{c|}{ Modelo } & NPAR & CMIN & DF & P & $\begin{array}{c}\text { CMIN/ } \\
\text { DF }\end{array}$ \\
\hline Ajuste do Mod. & 64 & 3376,19 & 371 & 0,000 & 9,100 \\
\hline CFI & \multicolumn{5}{|c}{0,853} \\
\hline RMSEA & \multicolumn{5}{|c}{085} \\
\hline
\end{tabular}

Fonte dados da pesquisa (2018).

O modelo retornou 9,100 para o valor da estatística $\mathrm{R}^{2}$ proporcional aos graus de liberdade e um valor RMSEA de 0,085, dentro dos limites previstos por Hair Jr. et al. (2009) para modelos com bom ajuste. $\mathrm{O}$ índice de ajuste comparativo do modelo foi de 0,853 , pouco abaixo do valor ideal de 0,900. A Tabela 5 apresenta os testes das hipóteses.

Nota-se que todas as hipóteses apresentadas no modelo foram validadas com estimativas positivas e com $p$-value significativos. Isso demonstra que, para a amostra de pesquisa utilizada nesta investigação, a Justiça Distributiva é fator determinante para a motivação e satisfação dos indivíduos da Geração Z nas organizações.

Tabela 5 Resultados dos testes das hipóteses

\begin{tabular}{l|l|c|l|l|l|l}
\hline Hipótese & \multicolumn{1}{|c|}{ Variável Dependente } & & \multicolumn{1}{|c|}{ Variável Independente } & Estimativa & \multicolumn{1}{c|}{ p valor } & \multicolumn{1}{c}{ Status } \\
\hline H1 & Justiça Distributiva & $\Rightarrow$ & Remuneração Estratégica & 1,069 & 0,000 & Aceita \\
\hline H2 & Justiça Distributiva & $\Rightarrow$ & Motivação & 0,515 & 0,000 & Aceita \\
\hline H3 & JD $>$ RE & $\Rightarrow$ & Motivação & 0,407 & 0,000 & Aceita \\
\hline H4 & Justiça Distributiva & $\Rightarrow$ & Satisfação & 0,299 & 0,000 & Aceita \\
\hline H5 & JD>RE & $\Rightarrow$ & Satisfação & 0,367 & 0,000 & Aceita \\
\hline H6 & Motivação & $\Rightarrow$ & Satisfação & 0,463 & 0,000 & Aceita \\
\hline
\end{tabular}

Fonte Dados da pesquisa (2018).

\subsection{Discussão dos resultados}

A partir dos resultados da pesquisa, entende-se que, para a categoria geracional Z, o desejo de liberdade, inovação e colaboração expostos por Tapscott (2010) se confirmam. O efeito da análise da pesquisa aponta que a Motivação e a Satisfação são influenciadas tanto pela Justiça Distributiva como pela Remuneração Estratégica.

Em consequência, os achados de Palozolli (2000), Assmar et al. (2005), Ahmad e Abdolkhalegh (2015), Sampaio et al. (2009), Tadeucci (2009) foram significativos pois os efeitos mostram que existe uma percepção da justiça distributiva e a mesma reflete na 
motivação para o trabalho na opinião dos indivíduos da categoria geracional $Z$, ao sentirem justiça e igualdade nos processos $(6,18)$ e nas recompensas $(6,16)$.

Quanto ao salário, os respondentes confirmam a importância sobre suas decisões e entendem que os variados tipos de remuneração estratégica, especialmente os de compartilhamento dos lucros ou bonificações, são relevantes, portanto, a provocação às empresas tange na definição da remuneração mais adequada a esse público, portanto os estudos de Trindad et al. (2011) também sancionam este estudo.

Nossos achados revelam que não é o tipo de remuneração que é o mais importante, mas a percepção de ganhos. Infere-se que entre as características de relacionamento, não quer ou não consegue lidar com ordens ou chefia $(5,18)$ não foi tão significativo quanto ter um bom relacionamento $(0,642)$, ou quanto a suas ideias serem ouvidas $(0,755)$. Evidentemente, o modelo de remuneração contribui para que desempenhem seu papel nas organizações com motivação e isso também afeta seu desejo de permanecer no cargo (LAZEAR, 1990).

A hipótese de que as percepções de justiça na avaliação de desempenho estão positivamente relacionadas com a satisfação no trabalho é condizente e ratifica os resultados de Colquitt (2001), Sotomayor (2007), Santos (2010) e Dalvesco et al. (2016). Destaca-se ainda estudos de Rego (2002) e Kim e Mauborgne (1997), cujas descobertas dão conta de que a justiça distributiva leva à satisfação com os resultados e consequentemente leva o indivíduo a cooperar também aparecem nesta pesquisa. Constatou-se também que a Justiça Distributiva tem impacto nas relações entre trabalhadores e organizações e a relação entre justiça e equidade influenciam nas atitudes e comportamentos neste ambiente, confirmando Assmar et al. (2005).

Pode-se afirmar ainda que os fatores motivacionais também estão presentes da forma como sugere-se nas hipóteses. Para Dawal, Taha e Ismail (2009) e Dal Vesco et al. (2016), o controle sobre dada tarefa proporciona maior satisfação, influencia na saúde, qualidade de vida e comportamento. Fatores como autonomia $(5,57)$, quando as ideias são ouvidas $(6,33)$ e também o reconhecimento pelo trabalho realizado $(6,46)$ estão presentes nos resultados, consequentemente, corroboram com os achados dos autores
(DAWAL; TAHA; ISMAIL, 2009; DAL VESCO et al., 2016).

A satisfação também é afetada pela motivação, além da justiça distributiva e da remuneração estratégica. Entende-se que os achados corroboram com Cavanagh (1992) e Dal Vesco et al. (2016) pois os indivíduos desempenham melhor seu trabalho quando estão satisfeitos. Consequentemente, a satisfação no trabalho resulta diretamente das percepções de justiça processual.

Por fim, os resultados percentuais da percepção dos indivíduos da Geração Z do oeste e meio oeste de Santa Catarina, somente confirmam que, apensar da categoria geracional $\mathrm{Z}$ estar mais atenta as tecnologias, ser imediatista, rápida, multitela, entre outros (VEIGA NETO et al., 2015), a relação existente entre a justiça distributiva, remuneração estratégica e os fatores de satisfação e motivação desses indivíduos torna-se fato assim como ocorreu nos demais desenhos teóricos citados neste estudo.

\section{CONCLUSÃO}

O estudo objetivou identificar a relação existente entre a justiça distributiva, a remuneração estratégica e os fatores de satisfação e motivação de indivíduos da Geração Z no mercado de trabalho. Para testar a relação, aplicou-se um questionário a 13.000 alunos de uma universidade, permanecendo uma amostra válida de 1.112 respondentes.

Este estudo contribui para a discussão sobre como fatores de recompensa organizacionais podem contribuir para a motivação e satisfação de colaboradores e, consequentemente, para melhoria nos resultados e no desempenho da empresa.

Sobre os resultados da pesquisa é importante destacar que embora a maioria dos pesquisados atuam na mesma área em que estudam, percebe-se que as remunerações recebidas variam de até 1 a 3 salários mínimos. Ou seja, é bem provável que se essa geração não for valorizada pelos seus esforços, salvo em períodos de grande recessão econômica que afeta sensivelmente o mercado de trabalho, irão trocar de emprego tão logo apareça uma oportunidade melhor.

Os resultados dessa pesquisa contribui para os gerentes pois descobrimos que alguns valores e 
características são consideradas importantes pelos indivíduos da geração $Z$, e que podem ser muito úteis para os gestores traçarem estratégias para a captação e retenção desses talentos. Dentre eles, observou-se que diferentes tipos de remuneração estratégica são importantes, pois o fator salarial tem influência na sua dedicação e na intenção de permanência no cargo. Valores como reconhecimento, boa comunicação e a percepção de justiça na distribuição de prêmios, salários ou mesmo punições e busca crescimento profissional e pessoal são considerados importantes no contexto dos pesquisados.

Cabe salientar ainda, que muitas áreas de atuação dos participantes da pesquisa não requer conhecimento especializado, situação que tende a mudar com a experiência profissional de cada um. Nesse ponto, caberá às empresas analisarem a relação custo $\mathrm{x}$ benefício para remunerar melhor alguns cargos em detrimento de outros, avaliando se os custos de remunerar melhor os colaboradores compensa mais que arcar com os custos da rotatividade oriundas da falta de motivação ou insatisfação no trabalho.

Quanto as contribuições científicas, separou-se em três contribuições. Pesquisas anteriores abordaram a justiça distributiva com foco na remuneração estratégica e como isso impacta no clima organizacional e satisfação dos colaboradores (ANDRADE, 2010; ODELIUS; SANTOS, 2008; AHMAD; ABDOLKHALEGH, 2015; OYADOMARI et al., 2009). Como mencionado anteriormente, analisar quais as condições favoráveis aos colaboradores para manterem-se nas organizações, associada com a inserção dos preceitos de justiça distributiva como forma de equalizar os retornos de acordo com a produtividade e desempenho dos colaboradores foi uma contribuição fundamental no presente estudo, pois validou-se a escala e confirmou-se a hipótese de que a justiça distributiva está positivamente relacionada à remuneração estratégica nas organizações, sendo esta a primeira contribuição.

A segunda contribuição enfatiza que a percepção da justiça distributiva afeta positivamente a motivação e que, por meio da remuneração estratégica, propicia sim motivação, ou seja, os membros da organização esperam que seus esforços sejam compensados de maneira justa, perante aos demais. Neste aspecto, corrobora-se com a literatura e validam-se a escala de motivação proposta (embasada em HERZBERG 1986; SAMPAIO et al., 2009; ASSMAR et al., 2005).

Por fim, a terceira contribuição à teoria se relaciona a justiça distributiva, a satisfação e motivação. Estudos anteriores (por exemplo REGO, 2002; KIM; MAUBORGNE, 1997) afirmam que a justiça distributiva leva à satisfação e consequentemente leva o indivíduo a cooperar. Seguindo com a literatura, constatou-se empiricamente que sim, as recompensas positivas são geram satisfação (TADEUCCI, 2009), ou seja, a percepção de justiça distributiva pelo empregado relaciona-se positivamente com a satisfação no trabalho, ao mesmo tempo, a motivação propicia satisfação.

Embora seja uma amostra representativa, os resultados não podem ser generalizados, pois a depender dos diferentes cenários, contexto, realidades ou mesmo cultura da região onde a pesquisa é aplicada, as percepções podem se diferir. Como sugestão de trabalhos futuros seria interessante acompanhar esses profissionais quando forem egressos dos seus cursos superiores, a fim de verificar a evolução desse estudo longitudinalmente. Também a comparação das percepções em diferentes organizações ou esferas públicas ou privadas poderia enriquecer a pesquisa e dar uma melhor dimensão dessas realidades.

\section{REFERÊNCIAS}

ANDRADE, S. M. Percepção de Justiça distributiva no clima organizacional - um estudo sobre organizações brasileiras que buscam se destacar pela qualidade do ambiente de trabalho. Dissertação de Mestrado, Universidade de São Paulo, USP, 2010.

ASSMAR, E. M. L.; FERREIRA, M. C.; SOUTO, S. O. Justiça organizacional: uma revisão crítica da literatura. Psicologia: reflexão e crítica, v. 18, n. 3, p. 443-453, 2005.

AZEVEDO, C. E. F.; OLIVEIRA, L. G. L., ABDALLA; M. M., GONZALEZ; R. K., RIBEIRO, A. J. G.; HOLPERIN, M. M. Por que finanças? Avaliando o interesse dos estudantes de graduação em administração pela área de finanças. Revista de Administração Mackenzie, v. 13, n. 6, p. 168-196, 2012. 
CERETTA, S. B.; FROEMMING, L. M. Geração Z: compreendendo os hábitos de consumo da geração emergente. Revista Eletrônica do Mestrado Profissional em Administração UNP, v. 3, n. 11, 2011.

COLQUIT, J. A. On dimensionality of organizational justice: A construct validation of measure. Journal of Applied Psicology, v. 86, n. 3, p. 368-400, 2001.

DAL VESCO, D. G.; BEUREN, I. M.; POPIK, F. Percepção de justiça na avaliação na avaliação de desempenho e satisfação do trabalho. Ref. Cont. UEM - Paraná, v. 35, n. 3, p. 121-138, setembro/ dezembro, 2016.

DAWAL, S. Z.; TAHA, Z.; ISMAIL, Z. Effect of job organization on job satisfaction among shop floor employees in automotive industries in Malaysia. International Journal of Industrial Ergonomics, v. 39 , p. 1-6, 2009.

EDWARD P. L. Why Is There Mandatory Retirement? Journal of Political Economy, v. 87, n. 6, p. 12611284, 1979.

FOLGER, R.; KONOVSKY, M. A. Effects of procedural and distributive justice on reactions to pay raise decisions. The Academy Management Journal, v. 32, p. 115-130, 1989.

GHENO, R.; BERLITZ, J. Remuneração estratégica e pacote de benefícios: um estudo de caso aplicado ao nível operacional de uma multinacional. Revista de Administração da UFSM, v. 4, n. 2, p. 268-287, 2011.

GOMIDE JUNIOR, S.; SIQUEIRA, M. M. M. Justiça no Trabalho. In: SIQUEIRA, M. M. M. (org.) Medidas do Comportamento Organizacional: Ferramentas de diagnóstico e gestão. Porto Alegre, Artmed, 2008.

HAIR JR., J.F. et al. Análise Multivariada de Dados. 5. ed. Porto Alegre: Bookman, 2005.
HAUSKNECHT, J. P., RODDA, J.; HOWARD, M. J. Targeted employee retention: Performance $\bigotimes$ based and job囚related differences in reported reasons for staying. Human Resource Management, v. 48, n. 2, p. 269-288, 2009.

HERZBERG, F. The motivation to work. 2. ed. Nova York: John Wiley e Sons, Inc. Indjejikian, R. J. (1999). Performance evaluation and compensation research: an agency perspective. Accounting Horizons, v. 13, n. 2, p. 147-157, 1964.

JACQUES, T. C. et al.. Geração Z: peculiaridades geracionais na cidade de Itabira-MG. RPCA - Revista Pensamento Contemporâneo em Administração, v. 9, n. 3, 2015.

A H M A D, K a m r a n ; G H O L A M I, Abdolkhalegh. Examine the relationship between organizational justice with job satisfaction and motivation and presenting some strategies to promote the sense of organizational justice in Refah bank in Kohgiluyeh and Byer-Ahmad." Advances in Environmental Biology, 2015, p. 346+. Academic OneFile, http:// link.galegroup.com/apps/doc/A417570392/ AONE $? \mathrm{u}=$ capes $\&$ sid $=$ AONE $\&$ xid $=40 \mathrm{bfdbb} 9$. Accessed 23 Nov. 2018.

LARKIN, I., PIERCE, L.; GINO, F. The psychological costs of pay-for-performance: implications for the strategic compensation of employees. Strategic Management Journal, v. 33, p. 1194-1214, 2012.

LAZEAR, E. P. Pensions and Deferred Benefits as Strategic Compensation. Industrial Relations, v. 29, n. 2, p. 263-280, 1990.

LAZEAR, E. P. Intergenerational externalities. Cambridge: National Bureau of Economic Research. 40 p., 1979.

MASLOW, A. H. Motivation and personality. Nova York: Harper e Row, 1954.

MCCRINDLE, M.; WOLFINGER, E. The ABC of XYZ: understanding the global generations. Sydney: UNSW Press, 2009. 
MERCHANT, K. A., VAN DER STEDE, W. A.; ZHENG, L. Disciplinary constraints on the advancement of knowledge: the case of organizational incentive systems. Accounting, Organizations and Society, v. 28, p. 251-286, 2003.

MURPHY, K. J. Executive compensation. In: Ashenfelter, O.; Card, D. (eds.), Handbook of labor economics, 3 p. 2485-2563, 1999.

NASCIMENTO, C., FRANCO, L. M. G.; CHERUBIN, A. P. M. S.. Associação entre remuneração variável e indicadores financeiros: evidências do setor elétrico. Revista Universo Contábil, v. 8, n. 1, p. 22-36, 2012.

NICHELE, J., STEFANO, S. R.; RAIFUR, L. Análise da remuneração estratégica para atrair e reter colaboradores: a visão dos pós-graduandos. Revista de Carreiras e Pessoas (ReCaPe), v. 5, n. 2, 2015.

NOVAES, M. V. A. Importância da motivação para o sucesso das equipes no contexto organizacional. Revista Eletrônica de Psicologia, v. 1, n. 1, p. 15-32, 2007.

ODELIUS, C. C.; SANTOS, A. R. Percepção de justiça organizacional de sistemas de remuneração em organizações públicas. Revista Alcance - Eletrônica, v. 15, n. 2. p. 226-242. 2008.

OYADOMARI, J. C. T. et al. Influências da Remuneração de Executivos na Congruência de Metas. Revista Contemporânea de Contabilidade, v. 1, n. 12, p. 53-74, 2009.

PALAIOLOGOS, A., PAPAZEKOS, P.; PANAYOTOPOULOU, L. Organizational justice and employee satisfaction in performance appraisal. Journal of European Industrial Training, v. 35, n. 8, p. 826-840, 2011.

PALAZOLLI, F. Percepção de Justiça nas Organizações como Antecedente dos comportamentos de cidadania organizacional. Caderno de Pesquisas em Administração, São Paulo, v. 7, p. 3, julho/setembro, 2000.
PAVLIK, E. L., SCOTT, T. W.; TIESSEN, P. Executive compensation: issues and research. Journal of Accounting Literature, v. 12, p. 131-189, 1993.

PRENDERGAST, C. The provision of incentives in firms. Journal of Economic Literature, v. 37, p. 7-63, 1999.

REGO, A. Justiça organizacional: Desenvolvimento e validação de um instrumento de medida. Psicologia, v. 14 , n. 2, 2000.

REIS NETO, M. T. A remuneração variável na percepção dos empregados e suas consequências na motivação e no desempenho. Tese (Doutorado em Administração) - Centro de PósGraduação e Pesquisas da Faculdade de Ciências Econômicas, Universidade Federal de Minas Gerais, Belo Horizonte, 2004.

SALVENDY, G. Effects of equitable and inequitable financial compensation on operator's productivity, satisfaction and motivation. The International Journal Of Production Research, v. 14, n. 2, p. 305310, 1976.

SAMPAIO, L. R., CAMINO, C. P. S.; ROAZZI, A. Justiça distributiva: uma revisão da literatura psicossocial e desenvolvimentista. Psicol Estud, v. 14, n. 4, p. 631-640, 2009.

TADEUCCI, M. S. R. Motivação e liderança. IESDE BRASIL AS, 2009.

TAPSCOTT, D. A hora da geração digital: como os jovens que cresceram usando a internet estão mudando tudo, das empresas aos governos. Rio de Janeiro: Agir Negócios, 2010.

TRINDADE, C., CUNHA, D. O., NUNES, N. M.; SANTOS, R. R. Até aonde os benefícios influenciam a permanência do funcionário na empresa? Revista CC Adm., v. 1, n. 1, 2011. 
VEIGA NETO, A. R., SOUZA, S. L. B. DE, ALMEIDA, S. T. DE, CASTRO, F. N.; BRAGA JUNIOR, S. S.. Fatores que influenciam os consumidores da Geração $\mathrm{Z}$ na compra de produtos eletrônicos. RACE, Revista de Administração, Contabilidade e Economia, v. 14, n. 1, 287-312, 2015.

ZAMBRANO, L. G., MERINO, J. D. G.; CASTELLANOS, A. R. Impacto de la inversión em capital humano sobre el valor empresarial. Academia, Revista latinoamericana de Adminstracíon, v. 51, p.15-26, 2012.

WOOD JR., T., PICARELLI FILHO, V. Remuneração estratégica: a nova vantagem competitiva ( $3 \mathrm{a}$ ed.). São Paulo: Editora Atlas, 2004. 